\title{
Diagnostic Étiologique Échographique des Douleurs Pelviennes Aigues de la Femme en Côte d'Ivoire
}

\author{
Ako-Ahui E, \\ Service de radiologie CHU de Treichville, Abidjan, Côte d'Ivoire \\ N'guessan KE, \\ Service de Gynécologie CHU de Treichville, Abidjan, Côte d'Ivoire \\ Konan AN, \\ Sétchéou A., \\ Ettien JJK, \\ Kouamé N., \\ Service de radiologie CHU de Yopougon, Abidjan, Côte d'Ivoire \\ N'goan-Domoua AM, \\ Hôpital Mère-Enfant de Bingerville,Abidjan, Côte d'Ivoire
}

Doi:10.19044/esj.2019.v15n21p437 URL:http://dx.doi.org/10.19044/esj.2019.v15n21p437

\section{Resume:}

Introduction : les douleurs pelviennes aigues chez la femme sont fréquentes et objet d'exploration échographique systématique dans notre pratique quotidienne. Objectif : contribuer à la recherche étiologique des douleurs pelviennes aigues (DPA) de la femme dans une zone où l'outil échographique est pratiquement le seul moyen d'imagerie accessible. Patiente et méthodes : étude prospective d'une durée de 6 mois réalisée au centre hospitalo-universitaire de Yopougon (Abidjan-Côte d'Ivoire). Une série continue de 153 femmes chez qui nous avons réalisé une échographie pelvienne au motif de DPA ont été enrôlées. Les examens échographiques ont été réalisées à l'aide de 3 sondes (convexe, linéaire et endocavitaire) en mode B et Doppler. Résultats : l'âge moyen des patientes était de 30, 2 ans avec des extrêmes de 12 et 68 ans. Les échographies étaient normales dans $25,5 \%$ et objectivaient une pathologie dans $74,5 \%$ des cas. Ces pathologies concernaient les organes génitaux internes dans $89,5 \%$ des cas et dans $10,5 \%$ des cas il s'agissait de pathologie extra-génitale. Les ovaires étaient les organes génitaux les plus concernés $(64,1 \%)$ suivis de l'utérus $(28,2 \%)$ et des trompes $(7,7 \%)$. Les causes extra-génitales étaient la hernie inguinale (4 cas), l'appendicite aigüe ( 5 cas), la sigmoïdite ( 1 cas) et la lithiase du bas uretère (2 cas). Conclusion : une proportion non négligeable de causes extra-génitales des douleurs pelviennes, objectivée dans notre étude, impose un examen 
clinique minutieux de toute la sphère hypogastrique voire abdominale par les cliniciens. L'utilisation de la sonde linéaire de haute fréquence qui n'est pas toujours sollicitée par les échographistes, au cours de la prise en charge des DPA devrait être préconisée.

Mots clés : Douleur pelvienne aigues, échographie pelvienne, Afrique

\title{
Ultrasound Findings in Acute Pelvic Pain in Women in Ivory Coast
}

\author{
Ako-Ahui E, \\ Service de radiologie CHU de Treichville, Abidjan, Côte d'Ivoire \\ N'guessan KE, \\ Service de Gynécologie CHU de Treichville, Abidjan, Côte d'Ivoire \\ Konan AN, \\ Sétchéou A., \\ Ettien JJK, \\ Kouamé N., \\ Service de radiologie CHU de Yopougon, Abidjan, Côte d'Ivoire \\ N'goan-Domoua AM, \\ Hôpital Mère-Enfant de Bingerville,Abidjan, Côte d'Ivoire
}

\begin{abstract}
Introduction: Acute pelvic pain in women is common and systematically explored by ultrasound in our daily practice. Objective: Contribute to the aetiological research of women's acute pelvic pain (APD) in an area where the ultrasound tool is practically the only means of accessible imagery. Patient and methods: A prospective study lasting 6 months was carrried out at the Yopougon University Hospital Center (Abidjan-Ivory Coast). A continuous series of 153 women who had a pelvic ultrasound for DPA were enrolled. Ultrasound examinations were performed using 3 probes (convex, linear and endocavitary) in B and Doppler mode. Results: The mean age of the patients was 30, 2 years with extremes of 12 and 68 years. About a quarter $(25.5 \%)$ of the ultrasound examinations were normal and in $74.5 \%$ of cases, a pathology was objectified. These pathologies concerned the internal genital organs in $89.5 \%$ of the cases and in $10.5 \%$ of cases they were extragenital pathologies. The ovaries were the most affected genitals (64.1\%)
\end{abstract}


followed by the uterus (28.2\%) and the fallopian tubes (7.7\%). Extra-genital causes were inguinal hernia (4 cases), acute appendicitis (5 cases), sigmoiditis ( 1 case) and lithiasis of the lower ureter (2 cases). Conclusion: a significant proportion of extragenital causes of pelvic pain, objectified in our study, requires a careful clinical examination of the whole hypogastric or even all abdominal regions by clinicians and the use of the high frequency linear probe which is not not always solicited by sonographers, during the care of the DPA.

Keywords: Acute pelvic pain, Pelvic ultrasound, Africa

\section{Introduction}

La douleur pelvienne aigue (DPA) de la femme constitue une situation fréquente. Elle est généralement le témoin d'une pathologie gynécologique mais elle peut être l'expression d'une affection digestive ou urinaire. En outre, elle peut constituer l'expression de conflits psychologiques somatisés dans cette zone pelvi-génitale (Poncelet, 2010 ; Fernandez 2005). De ce fait, la prise en charge de la DPA s'avère particulièrement difficile et doit être multidisciplinaire. Elle fait donc appel aux moyens d'imagerie médicale allant de l'échographie à l'imagerie par résonance magnétique (IRM) en passant par la tomodensitométrie (TDM). L'échographie se présente comme l'examen essentiel dans l'exploration de la douleur pelvienne chez la femme. C'est l'examen de première intention considérée comme le prolongement de l'examen gynécologique. Elle est souvent complétée par la TDM et l'IRM dans la recherche d'étiologies plus complexes telles que les torsions d'annexes, la pathologie endométriale, digestive et urinaire (Genevois, 2008). Dans notre contexte tropical où nous n'avons pas accès à la TDM ou à l'IRM soit par insuffisance du plateau technique ou par défaut de moyens financiers, quelle serait l'apport de l'échographie seule dans la prise en charge des douleurs pelviennes de la femme ? C'est à ce titre que nous nous sommes proposés de réaliser la présente étude dans le but de préciser l'apport réel et actuel de l'échographie dans le diagnostic étiologique des douleurs pelviennes de la femme dans une zone où le plateau technique et les moyens financiers limitent l'accès aux autres techniques d'imagerie médicale plus performantes.

\section{Patientes et Methodes}

Il s'est agi d'une étude prospective d'une durée de 6 mois allant d'octobre 2017 à avril 2018 réalisée au service de radiologie du CHU de Yopougon. Une série continue de cent cinquante-trois femmes chez qui nous avions réalisé une échographie pelvienne au motif de DPA, en dehors de toute notion d'aménorrhée gravidique connue, ont été enrôlées. Les examens échographiques avaient été réalisées à l'aide de 3 sondes (convexe, linéaire et endocavitaire) en mode B et Doppler. Les échographies ont été réalisées par 
des médecins échographistes séniors par voie sus pubienne à vessie pleine. La voie endovaginale, rarement systématique sauf à la demande du médecin traitant, venait en complément de la voie sus pubienne en cas de doute diagnostique. Il en était de même pour l'utilisation de la sonde linéaire qui servait à examiner l'environnement péri-génital lorsque la cause de la douleur n'était pas retrouvée au niveau de la sphère génitale. Les paramètres étudiés étaient l'âge, l'indication, le diagnostic d'organe et le diagnostic lésionnel. L'analyse statistique des résultats a été effectuée à l'aide du test de $\mathrm{Chi}^{2}$ de Pearson et du test exact de Fisher pour la comparaison des proportions. La comparaison des moyennes a été faite par le test de Student. Le seuil de significativité alpha a été fixé à $5 \%$.

\section{Resultats}

L'ensemble de nos résultats est résumé dans les tableaux I, II et III. L'âge moyen des patientes était de 30,2 ans avec des extrêmes de 12 et 68 ans. Les patientes de 25 à 35 ans étaient les plus nombreux avec une proportion de 43,8 \% des cas. La DPA isolée était le motif d'examen le plus fréquemment rencontré $(85,6 \%)$. Dans $7,8 \%$ des cas la DPA était accompagnée de dysménorrhée, de métrorragie dans $5,2 \%$ des cas et de masse pelvienne dans $1,3 \%$. Environ un quart $(25,5 \%)$ des échographies réalisées était normal et dans 74,5\% des cas, une pathologie avait été objectivée. Ces pathologies concernaient les organes génitaux internes dans $89,5 \%$ des cas et dans $10,5 \%$ des cas il s'agissait de pathologies extra-génitales. Les ovaires étaient les organes génitaux les plus concernés $(64,1 \%)$ suivis de l'utérus $(28,2 \%)$ et des trompes $(7,7 \%)$. Les lésions ovariennes étaient dominées par le syndrome des ovaires micropolykystiques $(84,4 \%)$ suivi des torsions de kystes $(12,5 \%)$ et des ovarites $(3,1 \%)$. La pathologie utérine était représentée par les myomes en nécrobiose $(72,4 \%$ des cas) suivis par des complications $(27,6 \%)$ de grossesse intra-utérine (décollement décidual, restes d'avortement, avortement en cours). Les lésions tubaires étaient dominées par les pyosalpinx $(88,9 \%)$ suivies des grossesses tubaires $(11,1 \%)$. Les causes extra-génitales étaient la hernie inguinale (4 cas), l'appendicite aigüe (5 cas), la sigmoïdite (1 cas) et la lithiase du bas uretère ( 2 cas). Sur le plan analytique, L'âge n'était pas significativement associé à la topographie lésionnelle. Le syndrome des ovaires micropolykystiques était observée chez des patientes relativement jeunes avec un âge de 22 ans ( $\mathrm{p}<0,001)$. Les myomes utérins étaient observés chez des patients relativement âgés avec un âge moyen de 35 ans $(\mathrm{p}=0,0063)$. 
Tableau I : Répartition des patientes en fonction de l'âge

\begin{tabular}{lll}
\hline CLASSE D'AGE & EFFECTIF & FREQUENCE (\%) \\
\hline$<15$ ans & 4 & 2,6 \\
$15-25$ & 40 & 26,1 \\
$25-35$ & 67 & 43,8 \\
$35-45$ & 35 & 22,9 \\
$45-55$ & 4 & 2,6 \\
$55-65$ & 2 & 1,3 \\
$>65$ ans & 1 & 0,7 \\
TOTAL & $\mathbf{1 5 3}$ & $\mathbf{1 0 0}$ \\
\hline
\end{tabular}

Tableau II : Répartition des patients selon les indications de l'échographie

\begin{tabular}{lcc}
\hline \multicolumn{1}{c}{ INDICATIONS } & EFFECTIF & FREQUENCE(\%) \\
\hline Pelvialgie & 130 & 85, \\
Pelvialgie (dysménorrhée) & 12 & 7,8 \\
Pelvialgie + métrorragie & 8 & 5,2 \\
Pelvialgie + masse pelvienne & 3 & 2 \\
TOTAL & 153 & 100 \\
\hline
\end{tabular}

Tableau III: Répartition des patientes en fonction du résultat de l'échographie

\begin{tabular}{|c|c|c|}
\hline DIAGNOSTIC ECHOGRAPHIQUE & EFFECTIF & FREQUENCE (\%) \\
\hline Echographie normale & 39 & 25,5 \\
\hline Echographie pathologique & 114 & $\mathbf{7 4 , 5}$ \\
\hline - Pathologie utérine & 29 & 25,4 \\
\hline - Nécrobiose (myome) & 21 & 18,4 \\
\hline - Complication de grossesse du T1 & 8 & 7 \\
\hline - Pathologie ovarienne & 64 & 56,1 \\
\hline $\begin{array}{ll}\text { - Syndrome des } \\
\text { micropolykystiques }\end{array}$ & 54 & 47,4 \\
\hline - $\quad$ Torsion de kystes & 8 & 7 \\
\hline - Ovarite & 2 & 1,7 \\
\hline - Pathologie tubaire & 9 & 7,9 \\
\hline - Pyosalpinx & 8 & 7 \\
\hline - $\quad \boldsymbol{G E} \boldsymbol{U}$ & 1 & 0,9 \\
\hline - Pathologie extra-digestive & 12 & 10,5 \\
\hline - Appendicite & 5 & 4,4 \\
\hline - Calcul jonction urétéro-vésical & 2 & 1,7 \\
\hline - $\quad$ Hernie inguinale & 4 & 3,5 \\
\hline - $\quad$ Sigmoïdite & 2 & 0,9 \\
\hline
\end{tabular}

\section{Discussion}

La DPA survient le plus souvent chez la femme en activité génitale (Genevois, 2008) comme le confirme notre étude dans lequel l'âge moyen des patientes était de 30, 2 ans et surtout la tranche d'âge dominante qui était celle entre 25 et 35 ans (43,8\%). Dans plus de $85 \%$ des cas cette douleur semblait être isolée, rendant ainsi difficile l'orientation diagnostique clinique. Ce qui confère à l'échographie un rôle primordial dans la recherche étiologique des 
DPA de la femme. Dans notre étude, l'échographie a été contributive dans $74,5 \%$ des cas. Dans environ $25,5 \%$ des cas de douleurs pelviennes, l'échographie s'est avérée normale. Ce résultat est proche de celui de Dellenbach et col (2001) qui estimaient qu'aucune lésion n'était retrouvée dans environ 30\% des douleurs pelviennes alors que Agoda et col (2011) a trouvé $52 \%$ de résultats normaux sur une série de 231 patientes au Togo sans utiliser la sonde linéaire. Il pourrait s'agir de douleurs pelviennes cycliques ou fonctionnelles ou de cause psychogène telles que décrites dans la littérature (Poncelet 2010 ; Fernandez 2005). Cependant, si l'échographie est la méthode de première intention dans l'exploration des douleurs pelviennes, d'autres moyens d'imageries plus performantes telles que l'IRM peuvent contribuer à baisser le taux de pelvialgies avec imagerie normale. Selon Genevois et col (2008), le radiologue doit savoir, en fonction du contexte clinique, quelle est la méthode d'imagerie la plus appropriée pour arriver au diagnostic étiologique. L'échographie est la première et parfois la seule investigation nécessaire. L'IRM est l'examen de choix du pelvis féminin et sera privilégiée chez la femme jeune ou enceinte. Le scanner trouve sa place quand l'origine gynécologique n'est pas cliniquement évidente ou dans le contexte infectieux du post-partum ou du postopératoire (Andreotti ; 2005). Dans notre contexte d'exercice, même si des appareils de scanners existent et que ceux d'IRM sont en plein essor, leur coût élevé renforce l'obligation d'utiliser le seul moyen accessible et peu onéreux qu'est l'échographie avec presqu'une obligation de résultats pour l'échographiste. Nous avons objectivé $74,6 \%$ de cas de pathologie dans notre étude, contribuant ainsi à une prise en charge efficace de notre patiente. Et ceci grâce à l'utilisation effective des trois sondes (convexe, linéaire et endovaginale). Notre pratique est conforme aux recommandations de la littérature. En effet pour Genevois et col (2008), il faut débuter l'exploration échographique de la douleur pelvienne par voie suspubienne avec éventuellement utilisation de la sonde de haute fréquence pour l'étude digestive, examen complété par la voie vaginale et l'étude Doppler si nécessaire.

Les étiologies de la douleur pelvienne de la femme sont multiples et peuvent se diviser en causes génitales et extra-génitales. Dans notre étude les causes géniales représentaient $89,5 \%$ cas. Elles étaient dominées par la pathologie ovarienne, elle-même dominée par le syndrome des ovaires micropolykystiques $(84,4 \%)$. Nos résultats concordent avec ceux d'Agoda et d'Adams (2011, 1986). Selon eux, la dystrophie ovarienne (figure 1) est la plus fréquente des maladies endocriniennes de la femme jeune. Elle touche plus de 5\% des femmes en âge de procréer (Shalev, 1998). Elle explique plus de 70\% des infertilités par anovulation (Adams, 1986 ; Carmina 1999). Selon la littérature, les causes utérines de douleur pelvienne sont la nécrobiose et les torsions de myomes. Si la nécrobiose a constitué $72,4 \%$ des cas rencontrés 
dans notre étude, nous n'avons pas objectivé des cas de torsion de myome sous-séreux pédiculé qui pourrait faire parti des cas normaux. Des complications de grossesse au début dont l'existence n'était pas connue par la patiente (notion d'absence de niveau scolaire pour la plupart des jeunes femmes en Afrique subsaharienne) ont constitué $26 \%$ des causes utérines.

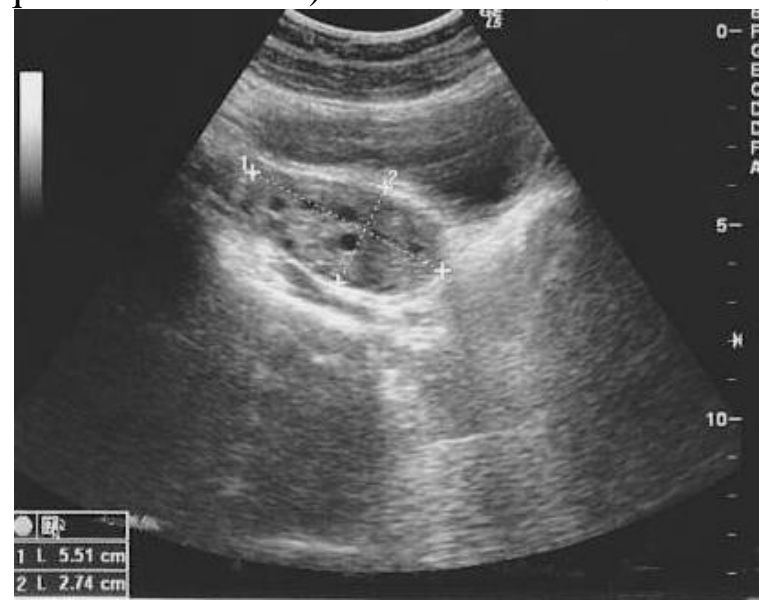

Figure 1. DPA chez une jeune femme de 21 ans. L'échographie réalisée par voie sus pubienne (sonde de 4Mhz) objective un ovaire droit mesurant $55 \mathrm{~mm}$ de longueur sur 27 $\mathrm{mm}$ d'épaisseur et $15 \mathrm{~cm}^{2}$ de surface. Son échostructure présente de nombreux microkystes et un stroma hypertrophique : syndrome des ovaires micropolykystiques

La pathologie tubaire était la plus impressionnante dans notre étude. Elle regroupait des processus infectieux pour la plupart et des grossesses ectopiques. Il s'agit selon la littérature de pathologie de la femme jeune en activité génitale porteuse de dispositif intra-utérin. Dans notre contexte, les infections sexuellement transmissibles (Carmina, 1999; Paik, 2006) sont les plus grandes pourvoyeuses d'abcès tubaire et leurs séquelles sont responsables des grossesses tubaires. Les signes échographiques de l'inflammation tubaire aigue ou chroniques sont connus. L'origine tubaire d'une pathologie est évoquée classiquement en échographie devant une masse siégeant entre utérus et ovaire, d'aspect tubulé, ayant une orientation en bas et en arrière, de calibre progressivement croissant, et présentant un aspect de pseudoplicature (figure 2). Le diamètre normal d'une trompe ne doit pas excéder $4 \mathrm{~mm}$ et classiquement, « une trompe visible est une trompe suspecte » (Randriamiarisoa, 2000). L'inflammation de la trompe conduit à un épaississement pariétal (classiquement $>5 \mathrm{~mm}$ ) et à un épaississement des franges tubaires. Lorsque la sonde est perpendiculaire à l'axe de la trompe, les franges tubaires apparaissent sous la forme d'une " roue dentée " ou " cogwhell sign ». Ce signe est considéré comme pathognomonique d'une inflammation tubaire aiguë (Randriamiarisoa, 2000). Lorsque la sonde est positionnée dans l'axe de la trompe, les franges tubaires prennent la forme de 
septa incomplets qui peuvent être retrouvés à la fois au stade aigu et chronique de l'infection (figure 2).

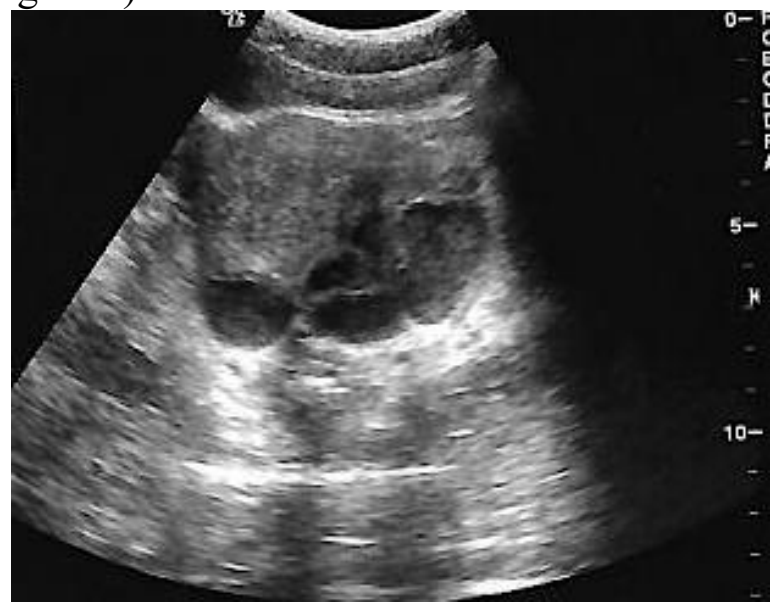

Figure 2. Jeune femme de 21 ans. DPA isolée. L'échographie réalisée par voie sus pubienne (sonde de $4 \mathrm{Mhz}$ ) objective un épanchement liquidien tubuliforme retro et latéro-utérin gauche d'allure septique comportant des cloisons incomplètes : pyosalpinx gauche

Il existe souvent un liquide inflammatoire péritubaire abondant en phase aiguë, moins abondant en phase chronique et une douleur élective au passage de la sonde dans le cul-de-sac vaginal postérieur. Une échographie pelvienne normale n'élimine en aucun cas le diagnostic qui sera fait à l'aide de prélèvements vaginaux (Vandermeer, 2009; Timor-Tritsch, 1998 ; Thomassin-Naggra 2008). Cependant l'urgence pelvienne à éliminer absolument en cas de douleur chez la femme jeune est la grossesse extrautérine (Timor-Tritsch 1998). Elle a constitué moins de 1\% des cas rencontrés dans notre étude. Un point d'honneur est à mettre sur les causes extra-génitales des douleurs pelviennes qu'il faut aller chercher lorsque l'échographie des organes génitaux internes est normale. La sonde linéaire de haute fréquence couplée ou non au mode Doppler prend ici toute son importance. Cette pathologie extra-digestive (figure 3) était dominée dans notre étude par la pathologie digestive (appendicite, hernie inguinale, sigmoïdite) à laquelle il faut compléter un cas de pathologie urinaire. Pour Pages-Bouic et col en France (2015), l'appendicite est la principale étiologie extra-génitale des douleurs pelviennes. Elle vient en première position parmi les étiologies objectivée dans notre étude. Mbongo et col au Congo (2016) ont mis en évidence 4 cas d'appendicite et 5 cas de pyélonéphrite aigue. La pathologie urinaire a été mise en évidence dans un seul cas. Il s'agissait d'une lithiase enclavée dans la jonction urétéro-vésicale. 


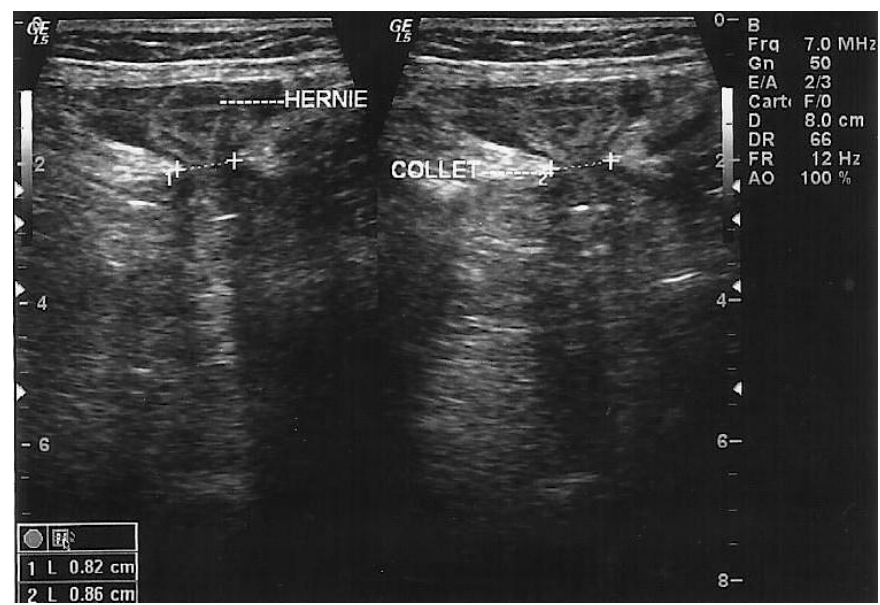

Figure 3. DPA aigue chez une femme de 47 ans. Echographie sus pubienne et endovaginale de la sphère génitale normale. L'examen de l'environnement péri-génital à l'aide de la sonde linéaire $(7 \mathrm{Mhz})$ objective une anse digestive inguinale incarcérée : hernie inguinale droite compliquée.

\section{Conclusion}

Les DPA de la femme, en Afrique subsaharienne, sont un motif de consultation fréquent et source d'une demande importante d'échographie. Elles surviennent fréquemment chez les femmes jeunes avec une moyenne d'âge de 30,2 ans en période d'activité génitale consultant essentiellement pour des pelvialgies isolées. Les ovaires sont les organes les plus concernés avec une forte proportion du syndrome des ovaires micropolykystiques. Les causes extra-génitales constituent $10,5 \%$ des étiologies. L'échographie pelvienne qu'elle soit pratiquée par voie sus pubienne ou par voie endovaginale représente l'outil paraclinique indispensable au diagnostic lésionnel et étiologique comme le démontre sa forte rentabilité diagnostique (74,5\% au cours de notre étude). Le quart de résultats normaux $(25,5 \%)$, considéré comme cause psychogène, devrait être réduit par la poursuite de l'exploration par des techniques d'imagerie plus performantes telles que la TDM et l'IRM. Dans le cas échéant, un examen clinique minutieux de toute la sphère hypogastrique voire abdominale par les cliniciens et l'utilisation de la sonde linéaire de haute fréquence qui n'est pas toujours sollicitée par les échographistes, est à souhaiter au cours de la prise en charge des DPA.

\section{Conflict d'internet}

Aucun

\section{Reference:}

1. Poncelet E, Bigot J, Hancart-Destée C, Ardaens Y. Imagerie des douleurs pelviennes. In Ardaens Y, du Masgenêt BG, Coquel P. 
Echographie et imagerie pelvienne en pratique gynécologique. 5th ed. Issy-les-Moulineaux: Elsevier Masson SAS; 2010. pp. 501-32.

2. Fernandez H. Douleurs pelviennes. EMC Médecine. 2005; 2: 341-56.

3. Genevois A, Marouteau N, Lemercier E, Dacher JN, Thiebot J. Imagerie de la douleur pelvienne aiguë. J radiol 2008 ; 89 (1-C2) : 92106.

4. Dellenbach P, Rempp C, Haering MT, Simon T, Magnier F, Meyer C. Douleur pelvienn chronique : une autre approche diagnostique et théraputique. Gynecol Obstet Fertil. 2001; 75: 234-43.

5. Agoda-koussema LK, Adama-Hondegla AB, Anoukoum T, Folligan K, Davi D, Awobanou K, N'dakena KG. Echographie sus pubienne dans les douleurs pelviennes chez la femme à Lomé. Journal Africain d'imagerie médicale 2011 ; (3) 6: 300-307.

6. Andreotti RF, Lee SI, Dejesus Allison SO, Bennett GL, Brown DL, Dubinsky $\mathrm{T}$ and al. ACR appropriateness Criteria Acute Pelvic pain in the reproductive age group Ultrasound $Q 2011 ; 27$ (3) : 205-210.

7. Adams J, Polson DW, Francks S. Prevalence of polycystic ovaries in women with anovulation and idiopathic hirsutism. Br Med J (Clin Res Ed) 1986; 293(6543): 355-9.

8. Shalev E, Yarom I, Bustan M, Weiner E, Ben-Shlomo I. Transvaginal sonography as the ultimate diagnostic tool for the management of ectopic pregnancy: experience with 840 cases. Fertil Steril. 1998; 69: 62-5.

9. Carmina E, Lobo RA. Polycystic ovary syndrome (PCOS): arguably the most common endocrinopathy is associated with significant morbidity in women. J clin Endocrinol Metab. 1999; 84(6): 1897-9.

10. Paik CK, Waetjen LE, Xing G, Dai J, Sweet RL. Hospitalizations for pelvic inflammatory disease and tuboovarian abscess Obstet Gynecol 2006 ; 107 (3) : 611-616.

11. Randriamiarisoa NAA, Andriamady RCL, Ranjalahy RJ, Rakotomanga S. Aspects épidémiologiques des algies pelviennes aiguës d'origine gynécologique à la Maternité du Centre Hospitalier de Befelatanana Antananarivo. Arch Inst Pasteur Madagascar 2000 ; $66(1 \& 2): 72-74$

12. Vandermeer FQ, Wong-You-Cheong JJ. Imaging of acute pelvic pain Clin Obstet Gynecol $2009 ; 52$ (1) : 2-20

13. Timor-Tritsch IE, Lerner JaviP, Monteagudo A, Murphy KE, Heller DS. Transvaginal sonographic markers of tubal inflammatory disease. Ultrasound Obstet Gynecol. 1998; 12: 56-66.

14. Thomassin-Naggra I, Dubernard G, Lafont C, Chopier J, Daraï E, Bazot M. Imagerie de l'infection pelviene. J Radiol. 2008; 89: 134-41. 
15. Pages-Bouic E, Millet I, Curros-Doyon F, Faget C, Fontaine M, Taourel P. Douleurs pelviennes aiguës en contexte septique et aseptique chez la femme. J Radiol 2015 ; 96 (5) : 401-12.

16. Mbongo JA, Foromo H, Otiobanda F, Iloki LH. Global Journal of Medical Research: E Gynecology and Obstetrics 2016 ;16(2) : 22-8. 